\title{
Random versus holographic fluctuations of the background metric. II. Note on the dark energies arising due to microstructure of space-time
}

\author{
Michael Maziashvili: \\ Andronikashvili Institute of Physics, 6 Tamarashvili St., Tbilisi 0177, Georgia \\ Center for Elementary Particle Physics, ITP, Ilia State University, 3-5 Cholokashvili Ave., Tbilisi 0162, Georgia
}

\begin{abstract}
Over the last few years a certain class of dark-energy models decaying inversely proportional to the square of the horizon distance emerged on the basis either of Heisenberg uncertainty relations or of the uncertainty relation between the four-volume and the cosmological constant. The very nature of these dark energies is understood to be the same, namely it is the energy of background space/metric uctuations. Putting together these uncertainty relations one nds that the model of random uctuations of the background metric is favored over the holographic one.
\end{abstract}

PACS numbers: 04.60.-m; 04.60.Bc

Keywords: Quantum gravity; Dark energy.

Space-time uncertainty is common for all approaches to quantum gravity. Quantum gravity strongly indicates the finite resolution of space-time; it will suffice to mention a few well known examples: space-time uncertainty relations in string theory [1, 2]; noncommutative space-time approach [3]; loop quantum gravity [4]; or space-time uncertainty relations coming from a simple Gedankenexperiments of space-time measurement [5]. Well known entropy bounds emerging via the merging of quantum theory and general relativity also imply finite space-time resolution [6]. The combination of quantum theory and general relativity in one or another way manifests that the conventional notion of distance breaks down at least at the Planck scale $l_{P} \simeq 10^{-33} \mathrm{~cm} \mathrm{[7].} \mathrm{Indeed,} \mathrm{the} \mathrm{finite} \mathrm{space-time} \mathrm{resolution} \mathrm{can}$ readily be shown in simple physical terms. (In what follows we will assume the system of units $\hbar=c=1$ ). Namely, posing the question as to what maximal precision one can mark a point in space by placing there a test particle, one notices that in the framework of quantum field theory the quantum takes up at least the volume, $\delta x^{3}$, defined by its Compton wavelength $\delta x \gtrsim 1 / m$. Not to collapse into a black hole, general relativity insists the quantum on taking up a finite amount of room defined by its gravitational radius $\delta x \gtrsim l_{P}^{2} m$. Combining together both quantum mechanical and general relativistic requirements one finds

$$
\delta x \gtrsim \max \left(m^{-1}, l_{P}^{2} m\right) .
$$

From this equation one sees that a quantum occupies at least the volume $\sim l_{P}^{3}$. Since our understanding of time is tightly related to the periodic motion along some length scale, this result implies in general the impossibility of space-time distance measurement to a better accuracy than $\sim l_{P}$. Therefore, the point in space-time can not be marked (measured) to a better accuracy than $\sim l_{P}^{4}$. It is tantamount to say that the space-time point undergoes fluctuations of the order of $\sim l_{P}^{4}$. Over the space-time region $l^{4}$ these local fluctuations add up in this or another way that results in four volume fluctuation of $l^{4}$. In view of the fact of how the local fluctuations of space-time add up over the macroscopic scale $\left(l \gg l_{P}\right)$, different scenarios come into play. Most interesting in quantum gravity are random and holographic fluctuations. If the local fluctuations, $\sim l_{P}$, are of random nature then over the length scale $l$ they add up as $\delta l=\left(l / l_{p}\right)^{1 / 2} l_{P}$. In the holographic case, the local fluctuations, $\sim l_{P}$, add up over the length scale $l$ to $\delta l=\left(l / l_{P}\right)^{1 / 3} l_{P} \cdot{ }^{1}$ Albeit locally (that is, at each point) the space-time undergoes fluctuations of the order of $\sim l_{P}^{4}$, for the fluctuations add up over the length scale $l$ to $\delta l(l)$, the region $l^{4}$ effectively looks as being made of cells $\delta l(l)^{4}$. Such a cell represents a minimal detectable unit of space-time over a region $l^{4}$ (in other words, it determines the maximum precision by which the volume $l^{4}$ can be known); that immediately prompts the rate of volume fluctuations $\delta V=\delta l(l)^{4}$. This is the quantity that enteres the uncertainty relation between the four volume and the cosmological constant.

Treating $\Lambda m_{P}^{2} / 8 \pi$ and four volume as conjugate to each other in the same sense as energy and time are conjugate in quantum mechanics, one can invoke the uncertainty principle to predict [8]

$$
\rho_{\Lambda} \simeq \frac{1}{\delta V}, \quad \text { where } \quad \rho_{\Lambda} \equiv \frac{\Lambda m_{P}^{2}}{8 \pi} .
$$

\footnotetext{
*Electronic address: maziashvili@gmail.com

${ }^{1}$ It is curious to notice that combining quantum mechanics with general relativity, the relation $\delta l \gtrsim l_{P}^{2 / 3} l^{1 / 3}$ as an intrinsic imprecision in measuring of length scale $l$ (for the Minkowskian background space) was obtained by Károlyházy in 1966 [5].
} 
So that the $\rho_{\Lambda}$ in Eq.(2) takes the definite meaning to be the local quantity

$$
\rho_{\Lambda} \simeq \frac{1}{\delta l^{4}} \text {. }
$$

In the case of random fluctuations, $\delta l \simeq\left(l_{P} l\right)^{1 / 2}$, from Eq.(3) one gets pretty good value of dark-energy density for the present epoch $l \simeq H_{0}^{-1}$ (where the present value of Hubble parameter $H_{0} \simeq 10^{-61} m_{P}$ ) [9]

$$
\rho_{\Lambda} \simeq \frac{1}{l_{P}^{2} l^{2}}
$$

However, we expect another dark energy to be also present simply because of time-energy uncertainty relation. Because of fluctuations, the length scale $l$ can be known with a maximum precision $\delta l$ determining thereby the minimal detectable cell $\delta l^{3}$ over the spatial region $l^{3}$. Such a cell represents the minimal detectable unit of space-time over a given length scale and if it has a finite age $t$, its existence due to time-energy uncertainty relation can not be justified with energy smaller then $\sim t^{-1}$. Hence, if the age of the background space-time is $t$ then because of time energy uncertainty relation the existence of minimal cell $\delta l^{3}$ over the region $l^{3}$ can not be justified with energy smaller than

$$
E_{\delta l^{3}} \gtrsim t^{-1} .
$$

Hence, time-energy uncertainty relation tells us that there should be another kind of dark energy [10]

$$
\rho \simeq \frac{E_{\delta l^{3}}}{\delta l^{3}}=\frac{1}{t \delta l^{3}} .
$$

For the Károlyházy uncertainty relation, $\delta l \simeq l_{P}^{2 / 3} l^{1 / 3}$, it gives

$$
\rho \simeq \frac{1}{l_{P}^{2} l t}
$$

which, by taking into account that during radiation and matter dominated epochs $t \simeq l$, is nothing else but Eq.(4) [10, 11].

However, from Eqs.(33,5) one sees that $\rho \ll \rho_{\Lambda}$ as long as $t \gg \delta l$. For holographic fluctuations

$$
\rho_{\Lambda} \simeq \frac{1}{\delta l^{4}} \simeq \frac{1}{l_{P}^{8 / 3} l^{4 / 3}},
$$

$\rho_{\Lambda}$ becomes unacceptably large $\rho_{\Lambda}\left(l \simeq 10^{61} l_{P}\right) \simeq 10^{-81} m_{P}^{4}$. On the other hand, for random fluctuations,

$$
\rho \simeq \frac{1}{t \delta l^{3}} \simeq \frac{1}{l_{P}^{3 / 2} l^{5 / 2}}
$$

$\rho$ is negligibly small in comparison with the observed value of dark energy density $\rho\left(l \simeq 10^{61} l_{P}\right) \simeq 10^{-152} m_{P}^{4}$.

The above discussion demonstrates that putting the four volume-cosmological constant uncertainty relation on the equal footing with the Heisenberg uncertainty relations [8], the model of random fluctuations appears to be favored over the holographic one. Curiously enough, the uncertainty relation $\delta l=\left(l_{P} l\right)^{1 / 2}$ is favored over the Károlyházy uncertainty relation, $\delta l=l_{P}^{2 / 3} l^{1 / 3}$, also in the framework of Gedankenexperiments for space-time measurement as well as in the context of quantum-gravitational running of space-time dimension [12]. It is important to keep in mind that the nature of both dark energies considered above is the same, namely it is the energy of background space/metric fluctuations.

The work was supported in part by the $C R D F / G R D F$ grant.

[1] G. Veneziano, Europhys. Lett. 2 (1986) 199;

D. J. Gross and P. F. Mende, Nucl. Phys. B303 (1988) 407;

D. Amati, M. Ciafaloni and G. Veneziano, Phys. Lett. B216 (1989) 41;

K. Konishi, G. Paffuti, P. Provero, Phys. Lett. B234 (1990) 276;

R. Guida, K. Konishi and P. Provero, Mod. Phys. Lett. A6 (1991) 1487. 
[2] M. Li and T. Yoneya, Phys. Rev. Lett. 78 (1997) 1219, hep-th/9611072

T. Yoneya, Prog. Theor. Phys. 103 (2000) 1081, hep-th/0004074 Prog. Theor. Phys. Suppl. 171 (2007) 87.

[3] D. Ahluwalia, Phys. Lett. B339 (1994) 301, gr-qc/9308007.

M. Maggiore, Phys. Lett. B304 (1993) 65, hep-th/9301067 Phys. Rev. D49 (1994) 5182, hep-th/9305163 Phys. Lett. B319 (1993) 83, hep-th/9309034

S. Doplicher, K. Fredenhagen and J. E. Roberts, Commun. Math. Phys. 172 (1995) 187, hep-th/0303037,

N. Seiberg and E. Witten, JHEP 9909 (1999) 032, hep-th/9908142.

[4] A. Ashtekar and J. Lewandowski, Class. Quant. Grav. 21, (2004) R53;

C. Rovelli, Quantum Gravity, (Cambridge University Press, Cambridge 2004);

T. Thiemann, Introduction to Modern Canonical Quantum General Relativity, (Cambridge University Press, Cambridge, (2007))

[5] F. Károlyházy, Nuovo Cim. A42 (1966) 390;

F. Károlyházy, A. Frenkel and B. Lukács, in Physics as Natural Philosophy (Eds. A. Shimony and H. Feschbach, MIT Press, Cambridge, MA, 1982);

F. Károlyházy, A. Frenkel and B. Lukács, in Quantum Concepts in Space and Time (Eds. R. Penrose and C. J. Isham, Clarendon Press, Oxford, 1986);

T. Padmanabhan, Class. Quant. Grav. 4 (1987) L107;

G. Amelino-Camelia, Mod. Phys. Lett. A9 (1994) 3415, gr-qc/9603014.

Y. J. Ng and H. van Dam, Mod. Phys. Lett. A9 (1994) 335;

F. Scardigli, Phys. Lett. B452 (1999) 39, hep-th/9904025.

R. Adler and D. Santiago, Mod. Phys. Lett. A14 (1999) 1371, gr-qc/9904026.

[6] J. D. Bekenstein, Phys. Rev. D23 (1981) 287; Phys. Rev. D49 (1994) 1912, gr-qc/9307035;

R. Bousso, JHEP 9907 (1999) 004, hep-th/9905177,

E. E. Flanagan, D. Marolf and R. M. Wald, Phys. Rev. D62 (2000) 084035, hep-th/9908070

R. Bousso, Rev. Mod. Phys. 74 (2002) 825, hep-th/0203101.

R. Bousso, E. E. Flanagan and D. Marolf, Phys. Rev. D68 (2003) 064001, hep-th/0305149

[7] C. Alden Mead, Phys. Rev. 135 (1964) B849; Phys. Rev. 143 (1966) 990;

L. J. Garay, Int. J. Mod. Phys. A10 (1995) 145, gr-qc/9403008.

[8] R. D. Sorkin, Int. J. Theor. Phys. 36 (1997) 2759, gr-qc/9706002 AIP Conf. Proc. 957 (2007) 142, arXiv: 0710.1675 [gr-qc].

[9] M. Ahmed, S. Dodelson, P. B. Greene and R. Sorkin, Phys. Rev. D69 (2004) 103523, astro-ph/0209274;

X. Calmet, Europhys. Lett. 77 (2007) 19902, hep-th/0510165;

T. Padmanabhan, Class. Quant. Grav. 19 (2002) L167, gr-qc/0204020 Class. Quant. Grav. 19 (2002) 3551, gr-qc/0110046.

[10] N. Sasakura, Prog. Theor. Phys. 102 (1999) 169, hep-th/9903146

M. Maziashvili, Int. J. Mod. Phys. D16 (2007) 1531, gr-qc/0612110

[11] M. Maziashvili, Phys. Lett. B652 (2007) 165, arXiv: 0705.0924 [gr-qc];

R. G. Cai, Phys. Lett. B657 (2007) 228, arXiv: 0707.4049 [hep-th];

H. Wei and R. G. Cai, Phys. Lett. B660 (2008) 113, arXiv: 0708.0884 [astro-ph].

[12] M. Maziashvili, Int. J. Mod. Phys. A23 (2008) 1747, arXiv: 0709.0898 [gr-qc];

M. Maziashvili, Phys. Lett. B679 (2009) 482, arXiv: 0809.5006 [gr-qc]. 